\title{
Natural Product Piperine Alleviates Experimental Allergic Encephalomyelitis in Mice by Targeting Dihydroorotate Dehydrogenase
}

zehui liu ${ }^{1}$, qian $\mathrm{hu}^{2}$, wanyan wang ${ }^{2}$, sisi lu ${ }^{2}$, Dang $\mathrm{Wu}^{3}$, shuyin ze ${ }^{4}$, Jiacheng $\mathrm{He}^{5}$, Ying huang ${ }^{6}$, wuyan chen ${ }^{7}$, Yechun $\mathrm{Xu}^{8}$, Weiqiang $\mathrm{Lu}^{5}$, and Jin Huang ${ }^{3}$

${ }^{1}$ East China University of Science and Technology, School of Pharmacy Shanghai, CN

${ }^{2}$ Shanghai Key Laboratory of New Drug Design, School of Pharmacy, East China

University of Science and Technology, Shanghai, China

${ }^{3}$ East China University of Science and Technology

${ }^{4}$ Shanghai Key Laboratory of Regulatory Biology, Institute of Biomedical Sciences and School of Life Sciences, East China Normal University, Shanghai, China

${ }^{5}$ East China Normal University

${ }^{6}$ Guangdong Institute for Drug Control, Guangzhou, Guangdong, China

${ }^{7}$ CAS Key Laboratory of Receptor Research, Shanghai Institute of Materia Medica, Chinese Academy of Sciences (CAS), Shanghai, China

${ }^{8}$ Shanghai Institute of Materia Medica Chinese Academy of Sciences

May 5, 2020

\begin{abstract}
BACKGROUND AND PURPOSE Human dihydroorotate dehydrogenase (DHODH) represents a promising therapeutic target for chronic inflammatory and autoimmune diseases. The aim of this study was to discover novel DHODH inhibitor and evaluate the potential of DHODH inhibition in treating multiple sclerosis (MS), a popular chronic inflammatory disease of the central nervous system. EXPERIMENTAL APPROACH Biochemical and Biophysical methods, including enzymatic kinetic analysis, thermofluor assay, isothermal titration calorimetry and X-ray crystallography were used to assess DHODH inhibition. The immunomodulatory activity was assessed by using concanavalin a-triggered T-cell assay and mixed lymphocyte reaction assay. MOG-induced experimental allergic encephalomyelitis (EAE) was used to assess the in vivo therapeutic effects. Myelin destruction and blood-brain barrier (BBB) was evaluated via in vivo imaging KEY RESULTS Piperine was identified as a natural inhibitor of human DHODH with an IC50 value of $0.88 \pm 0.04 \mu \mathrm{M}$. In addition, we resolved the co-complex crystal structure of DHODH and piperine at $1.98 \AA$ resolution and found that Tyr356 residue of DHODH is critical for piperine binding. Moreover, piperine can markedly suppress $\mathrm{T}$ cell overactivation via a DHODH dependent-manner. Finally, we found that piperine exhibits strong preventive and therapeutic effect in the MOG-induced EAE by restricting inflammatory cells infiltration into the CNS and by preventing myelin destruction and blood-brain barrier (BBB) disruption. CONCLUSION AND IMPLICATIONS Taken together, these findings highlight DHODH as a therapeutic target for autoimmune disease of the nervous system, and demonstrate a novel pharmacological role for piperine in the treatment of MS.
\end{abstract}

\section{EXPERIMENTAL APPROACH}

Biochemical and Biophysical methods, including enzymatic kinetic analysis, thermofluor assay, isothermal titration calorimetry and X-ray crystallography were used to assess DHODH inhibition. The immunomodulatory activity was assessed by using concanavalin a-triggered T-cell assay and mixed lymphocyte reaction 
assay. MOG-induced experimental allergic encephalomyelitis (EAE) was used to assess thein vivo therapeutic effects. Myelin destruction and blood-brain barrier (BBB) was evaluated via in vivo imaging

\section{KEY RESULTS}

Piperine was identified as a natural inhibitor of human $\mathrm{DHODH}$ with an $\mathrm{IC}_{50}$ value of $0.88 \pm 0.04 \mu \mathrm{M}$. In addition, we resolved the co-complex crystal structure of DHODH and piperine at $1.98 \AA$ resolution and found that Tyr356 residue of DHODH is critical for piperine binding. Moreover, piperine can markedly suppress $\mathrm{T}$ cell overactivation via a DHODH dependent-manner. Finally, we found that piperine exhibits strong preventive and therapeutic effect in the MOG-induced EAE by restricting inflammatory cells infiltration into the CNS and by preventing myelin destruction and blood-brain barrier (BBB) disruption.

\section{CONCLUSION AND IMPLICATIONS}

Taken together, these findings highlight DHODH as a therapeutic target for autoimmune disease of the nervous system, and demonstrate a novel pharmacological role for piperine in the treatment of MS.

\section{Abbreviations}

BBB, blood-brain barrier; CD, circular dichroism; CNS: central nervous system; CoQ0, Coenzyme Q0; DCIP: 2, 6-dichloroindophenol; DHO: dihydroorotate; DHODH, dihydroorotate dehydrogenase; FMN, flavin mononucleotide; ITC, isothermal titration calorimetry; MS, multiple sclerosis;

\section{Introduction}

Multiple sclerosis (MS) is a chronic inflammatory demyelinating disease of the central nervous system (CNS) that afflicts approximately 2.5 million people worldwide(Baecher-Allan, Kaskow \& Weiner, 2018; Goverman, 2009). MS lesions can emerge in the whole CNS and are most easily observed in the white matter as focal regions of inflammation and demyelination(Reich, Lucchinetti \& Calabresi, 2018). The well-defined cause of MS still remains elusive now, although genetic susceptibility and environmental factors have been associated with the MS occurrence(Ascherio, 2013; Dendrou, Fugger \& Friese, 2015a). MS is the leading cause of disability in young adults, resulting in serious socioeconomic consequences for the individual patient and for society (approximately $\$ 10$ billion in United States)(Adelman, Rane \& Villa, 2013; Holland, Schneider, Rapp \& Kalb, 2011; Jennum, Wanscher, Frederiksen \& Kjellberg, 2012). Therefore, development of innovative therapeutic is an urgent need to improve the clinical benefit and life quality for MS patients.

MS is widely considered to be an inflammatory T-cell-mediated autoimmune disease of CNS(Ridderstad Wollberg et al., 2014). In clinical MS and EAE model, immune system T leukocytes transmigrate from the bloodstream into the CNS to assault the myelin sheath surrounding nerve fibers(Zozulya \& Wiendl, 2008). Autoreactive T cells indiscriminately recognize CNS antigens play a critical pathogenic role in the induction of CNS inflammation and demyelination, and thereby in the progression of neurological function deficit and physical disability(T Hart \& Massacesi, 2009). In clinical, increased population of activated T cells against the myelin were observed in MS patients compared to healthy human. Autoreactive $\mathrm{CD} 4^{+} \mathrm{T}$ cells were considered as the key driver events in EAE models, indicating important immunopathologic features of MS(Dendrou, Fugger \& Friese, 2015a). Currently, increasing animal experiment results and clinical trials data of MS demonstrate that CD8 ${ }^{+}$T-cells may also contribute to inflammation sustaining and tissue destruction in MS(Friese \& Fugger, 2005; Lassmann \& Bradl, 2017). Collectively, T lymphocytes have emerged as important players in the pathogenic mechanism of CNS autoimmune inflammation, representing a promising therapeutic target of MS.

Human Dihydroorotate dehydrogenase (DHODH) is a rate-limiting enzyme in the de novo pyrimidine biosynthesis pathway. DHODH binds to flavin mononucleotide (FMN) cofactor in conjunction with ubiquinone to catalyze the oxidation of dihydroorotate to orotate(Löffler, Jöckel \& Schuster, 1997; Munier-Lehmann, Vidalain, Tangy \& Janin, 2013; Sykes et al., 2016). Human DHODH is located in the intracellular mitochondrial inner membrane and thereby can be categorized into class 2 DHODH family. As an essential protein that catalyzes the conversion of DHO to orotate, DHODH is a critical player in the pyrimidine de novo bio- 
synthesis of DNA and RNA. In high proliferative cells, such as activated T lymphocytes, increased de novo pyrimidine biosynthesis can confer their superior growth capacity(Quemeneur, Gerland, Flacher, Ffrench, Revillard \& Genestier, 2003). Blockade of de novo pyrimidine biosynthesis by pharmacological targeting of DHODH has been considered as a promising therapeutic strategy for autoimmune diseases through inhibition of T lymphocytes activation. Teriflunomide (A77 1726) have been approved for the treatment of MS through blocking pyrimidine de novobiosynthesis at the level of DHODH(Dimitrova, Skapenko, Herrmann, Schleyerbach, Kalden \& Schulze-Koops, 2002; Ruckemann et al., 1998). While, its observed hepatotoxicity in clinical and potential teratogenicity in preclinical model limit its benefit.

In the present study, we identified piperine, a main bioactive constituent of traditional medicine black pepper, as a natural inhibitor of human DHODH using a panel of biochemical and biophysical methods, including enzymatic assay, isothermal titration calorimetry and X-ray crystal diffraction. We further demonstrated that DHODH inhibition by piperine ameliorated MOG-induced EAE mouse model accompanied with reduced inflammation as well as lessened myelin and BBB destruction. Taken together, our data revealed that piperine targets $\mathrm{T}$ cells in EAE by inhibition of DHODH and provided a potential treatment strategy for MS patients.

\section{Materials and Methods}

\section{Reagents}

Piperine and A771726 were obtained from Sigma-Aldrich (Shanghai, China). Fluorescent probe BSA-Cy5.5 and DBT were purchased from Biolead Biology Sci\&Tech (Beijing, China). MOG35-55 peptide was obtained from GL Biochem (Shanghai, China). Antibodies against mouse CD4 (Product \# 17-0041-81) and CD8 (Product \# 11-0081-81) were purchased from ebioscience (CA, USA). All other reagents were purchased from Sigma-Aldrich unless otherwise indicated.

\section{Protein expression and purification}

Human DHODH protein expression and purification were performed as described in our previous studies(Wu et al., 2018).

\section{DHODH inhibition Assay}

DHODH enzymatic activity was determined using 2, 6-dichloroindophenol (DCIP) method as previously described(Diao et al., 2012). In brief, recombinant human DHODH (15 nM) was incubated with varied concentrations of indicated compounds for $10 \mathrm{~min}$ at room temperature. The assay buffer was composed of $50 \mathrm{mM}$ Tris (pH 8.0), $120 \mu \mathrm{M}$ DCIP, $100 \mu \mathrm{M}$ Coenzyme Q0 (CoQ0), 0.1\% Triton X-100. The enzymatic reaction was initiated by adding dihydroorotate (DHO) with a final concentration of $500 \mu \mathrm{M}$ and absorption at $600 \mathrm{~nm}$ was continuously recorded for 6 min with EnVision Multimode Plate Reader (Perkin Elmer, Waltham, MA). The $\mathrm{IC}_{50}$ values were calculated by GraphPad Prism 7 (GraphPad software, CA).

\section{Fluorescence-based thermal shift assay}

Briefly, SYPRO orange stock solution (Invitrogen, Carlsbad NY) was diluted 1:1000 into the assay buffer (25 $\mathrm{mM}$ Hepes, $150 \mathrm{mM} \mathrm{NaCl}, \mathrm{pH} 7.5)$, and purified $\mathrm{DHODH}$ was added with a final concentration of $5 \mu \mathrm{M}(\mathrm{Lu}$ et al., 2017). Then, the mixture $(40 \mu \mathrm{L})$ was loaded into the 96 -well iCycler iQ PCR plate (Bio-Rad, CA, USA) with different compounds $(50 \mu \mathrm{M})$. The plate was heated from 25 to $80{ }^{\circ} \mathrm{C}$ with a rate of $0.50{ }^{\circ} \mathrm{C} / \mathrm{min}$ and the fluorescence intensity was measured at $\mathrm{Ex} / \mathrm{Em}=490 / 530 \mathrm{~nm}$ using a $\mathrm{CF} \times 96 \mathrm{TM}$ Real Time System (Bio-Rad, Hercules, CA) according to the manufacturer's instructions.

\section{Enzymatic kinetic analysis}

Lineweaver-Burk analysis was carried out as described previously(McLean, Neidhardt, Grossman \& Hedstrom, 2001). Briefly, a variety of concentrations of CoQ $(25-200 \mu \mathrm{M})$, DHO $(25-200 \mu \mathrm{M})$ and compound $(1-3 \mu \mathrm{M})$ were incubated with DHODH $(20 \mathrm{nM})$ in reaction buffer $(50 \mathrm{mM}$ Tris, $\mathrm{pH} 8.0$, and $150 \mathrm{mM} \mathrm{KCl})$. The enzymatic reaction was detected at $287 \mathrm{~nm}$ using a Hitachi U-2000 spectrophotometer (Tokyo, Japan). 
In addition, kinetic curves of DHODH activity in the presence of varied concentrations of PIP was generated to calculate the $\mathrm{K}_{\mathrm{on}}$ and $\mathrm{K}_{\mathrm{off}}$ values. All experiments were performed at $25{ }^{\circ} \mathrm{C}$. The experiments were performed at least three times.

\section{Isothermal titration calorimetry (ITC)}

Thermodynamic characterization of DHODH-PIP interactions was performed using an iTC200 instrument (Microcal, GE Healthcare, PA, USA) as described before ${ }^{2}$,(Zhao et al., 2015). Before titrations, the stock solutions of PIP and DHODH protein were diluted with ITC buffer $(50 \mathrm{mM}$ HEPES, $300 \mathrm{mM} \mathrm{NaCl}, 10 \%$ Glycerol, $0.1 \%$ Triton) at the concentrations of $100 \mu \mathrm{M}$ and $20 \mu \mathrm{M}$, respectively. All titrations of PIP into DHODH were performed by 19 identical injections of $2 \mu \mathrm{l}$ with a duration of 4 seconds per injection spaced at interval of 120 seconds between injections following an initial injection of $0.4 \mu \mathrm{l}$ at $25{ }^{\circ} \mathrm{C}$. The reaction heat of injecting PIP into the DHODH were obtained and the data was processed by the supplied MicroCal Origin software package (PA, USA). The final concentration of DMSO in the assay buffer is less than $3 \%$. All the calorimetric data were calculated with the formula $\Delta \mathrm{G}=\Delta \mathrm{H}-\mathrm{T} \Delta \mathrm{S}=-\mathrm{RT} \ln \mathrm{K}$, where $\mathrm{T}$ is experimental temperature, $\mathrm{R}$ is gas constant, $\mathrm{K}$ is binding constant, $\Delta \mathrm{G}$ represents changes of free energy, $\Delta \mathrm{H}$ represents changes of enthalpy and $\Delta \mathrm{S}$ represents changes of entropy of, respectively.

\section{Fluorescence quenching assay}

Fluorescence quenching analysis was performed as previously described(Xu et al., 2015). The purified DHODH protein was incubated with various concentrations of PIP at $4{ }^{\circ} \mathrm{C}$ for $1 \mathrm{~h}$ and fluorescence spectra were detected using a Cary Eclipse fluorescence spectrophotometer (Agilent Technologies, CA, USA). Excitation wavelength was set at $280 \mathrm{~nm}$ and emission spectra ranging from 285 to $600 \mathrm{~nm}$ was recorded.

\section{CD spectroscopy}

Briefly, DHODH protein were dialyzed against $10 \mathrm{mM}$ phosphate buffer (pH 7.4) and incubated with different concentrations of PIP for 2 min at room temperature. The circular dichroism (CD) spectra were measured by chirascan circular dichroism spectrometer (Applied PhotoPhysics, United Kingdom) in a quartz cell with a 0.5 -cm path length.

\section{Crystallization ofDHODH-PIP complexes}

Optimal crystals were grown by hanging drop vapor diffusion method at $20 \mathrm{degC}$ as previously reported(Liu, Neidhardt, Grossman, Ocain \& Clardy, 2000b). Drops were prepared by mixing $1.0 \mu \mathrm{L}$ of $20 \mathrm{mg} / \mathrm{ml}$ DHODH protein solution which contained $2 \mathrm{mM}$ PIP, $50 \mathrm{mM}$ HEPES pH 7.7, $400 \mathrm{mM} \mathrm{NaCl}, 30 \%$ Glycerol, $1 \mathrm{mM}$ EDTA, $10 \mathrm{mM}$ (N, N-dimethylundecylamine-N-oxide) UDAO, and $20.8 \mathrm{mM} \mathrm{N}$, N-dimethyldecylamine- Noxide (DDAO) with equal-volume precipitant solution of $0.1 \mathrm{M}$ acetate, $\mathrm{pH} 4.8$, and 2.4-2.6 $\mathrm{M}$ ammonium sulfate. The drops were incubated against $0.5 \mathrm{~mL}$ of $50 \mathrm{mM}$ HEPES pH 7.7, $300 \mathrm{mM} \mathrm{NaCl}, 20 \%$ Glycerol, 1 $\mathrm{mM}$ EDTA, $10 \mathrm{mM}$ UDAO and $20.8 \mathrm{mM}$ DDAO. Diffraction-quality crystals were appeared usually in three weeks and reached the full size of $0.15 \times 0.15 \times 0.153 \mathrm{~mm}$. Crystals were flash frozen in liquid nitrogen for data collection.

\section{Data collection and structure determination}

X-ray diffraction data were collected at the synchrotron beamline BL19U1 in SSRF, Shanghai, China. The crystals were maintained at $100 \mathrm{~K}$ during data collection. Data were indexed, integrated and scaled using HKL3000 (HKL Research, Charlottesville, VA). The structure of the complex was determined by molecular replacement using molrep (CCP4) and PDB entry 4JTU as an initial model. The resulting model was rebuilded and refined in Coot. The images were generated using the software PyMOL (Molecular Graphics System, Version 2.2.3; Schrödinger, LLC). A summary of the data collection and processing statistics was given in Table S1. The coordinates and structure factors of the final model of DHODH-piprine were deposited in the Protein Data Bank (PDB, www.rcsb.org, PDB ID: 6IWU).

\section{Mouse spleen cell proliferation}


Single-cell suspensions of spleens from individual C57BL/6 mice were harvested as described previously(Wanke et al., 2017). Briefly, the spleen was aseptically taken from mice, cut into small pieces and crush gently through the cell strainers in $5 \mathrm{ml} 1 \times$ HBSS. Cells were suspended in Ammonium-Chloride-Potassium (ACK) lysing buffer to remove the erythrocytes and then collected by centrifugation at $2000 \mathrm{rpm}$ for $10 \mathrm{~min}$. After washing twice with $1 \times$ HBSS, cells were re-suspended in RPMI-1640 with $10 \%$ FBS for culture. Spleen cells were seeded into 96 -wells cell culture plates at a density of $5 \times 10^{5}$ cells/well in RPMI-1640 with $10 \%$ FBS and subsequently incubated with various concentrations of PIP in the presence or absence of uridine $(100 \mu \mathrm{M})$. Cell proliferation was stimulated by adding $10 \mu \mathrm{g} / \mathrm{ml}$ of Con A. After $72 \mathrm{~h}$ treatment, cells growth was measured by MTT assay with EnVision Multimode Plate Reader (Perkin Elmer, Waltham, MA).

\section{Mixed lymphocyte reaction (MLR) assay}

The two-way mouse mixed lymphocyte reaction was conducted as described in our previous study(Diao et al., 2012). In brief, single-cell suspensions of spleens from C57BL/6 and BALB/C were prepared as foregoing mentioned. Each set of $2 \times 10^{5}$ cells were seed into 96-wells plates in RPMI-1640 with $10 \%$ FBS and $50 \mu \mathrm{M} \beta$-mercaptoethanol. Cells were incubated for 4 days with indicated concentrations of PIP in the presence or absence of uridine $(100 \mu \mathrm{M})$. Cell proliferation was evaluated by MTT as described above and the proliferation of BALB/C spleen cells alone was served as background.

\section{DHODH activity in Jurkat cells}

Intracellular DHODH enzymatic activities was analyzed as described before(Yin, Kabashima, Zhu, Shibata \& Kai, 2017). Jurkat T cells were cultured in RPMI-1640 with $10 \%$ FBS and treated with different concentrations of PIP. After $24 \mathrm{~h}$ incubation, cells were harvested by centrifugation and lysed in NP-40 lysis buffer at $4^{\circ} \mathrm{C}$ for $15 \mathrm{~min}$. Lysates were clarified at $12000 \mathrm{rpm}$ for $10 \mathrm{~min}$ and the subsequent supernatant was used in DHODH reaction. Lysate $(200 \mu \mathrm{L}$ ) was incubated in buffer A (the final volume, $1.0 \mathrm{~mL}$ ), containing 500 $\mathrm{mM}$ DHO, $200 \mathrm{mM} \mathrm{K} \mathrm{CO}_{3}-\mathrm{HCl}\left(\mathrm{pH} 8.0\right.$ ), 0.2 Triton $\mathrm{X}-100$, and $100 \mu \mathrm{M}$ CoQ0 at $37^{\circ} \mathrm{C}$ for $1 \mathrm{~h}$. Mixture of enzyme reaction $(200 \mu \mathrm{L})$ was mixed with buffer B, containing $1 \mathrm{mM} 4$-TFMBAO, $2 \mathrm{mM} \mathrm{K} 3[\mathrm{Fe}(\mathrm{CN}) 6]$, and $20 \mathrm{mM} \mathrm{K}_{2} \mathrm{CO}_{3}$ and then heated at $80{ }^{\circ} \mathrm{C}$ for $5 \mathrm{~min}$. The reaction was stopped by cooling in ice and the fluorescence signal was recorded using a PT1-QM4 steady-stead fluorimeter (PTI, USA) at excitation and emission wavelengths of 340 and $460 \mathrm{~nm}$, respectively.

\section{$\mathrm{MOG}_{35-55}$ induced chronic progressive EAE model in C57BL/6 mice}

10-12 weeks old female C57BL/6 mice were purchased from Shanghai Laboratory Animal Center. All animal care and experimental procedures in this study were conducted in compliance with the protocol approved by the institutional Animal Care and Use Committee at East China University of Science and Technology. Mice were housed for 7 days in a flow cabinet with a $12 \mathrm{~h}$ light/dark cycle and fed with standard rodent chow and tap water ad libitum. For EAE induction, mice were immunized in the flank with $100 \mu \mathrm{g}$ MOG35-55 peptide dissolved in Complete Freund's Adjuvant (CFA, including extract $4 \mathrm{mg} / \mathrm{ml}$ M. tuberculosis H37Ra; Beijing Biolead Biology Sci \& Tech)(Najm et al., 2015). The $200 \mathrm{ng} /$ mouse pertussis toxin (PTX) was injected on day 0 and day 2. Treatment with PIP or vehicle started after immunization after 12 days and kept 17 days. Clinical symptoms scoring for MOG35-55 induced EAE was evaluated as follows: score 0, no obvious changes in motor functions; score 0.5, distal paralysis of the tail; score 1, complete tail paralysis; score 1.5, mild paresis of one or both hind legs; score 2, severe paresis of hind legs; score 2.5, complete paralysis of one hind leg; score 3, complete paralysis of both hind legs.

\section{Flow Cytometry Analysis}

Preparation of individual cell suspensions from EAE mouse was performed as described before. Splenocytes were resuspended in FACS buffer (PBS, pH7.4, supplemented with $1 \mathrm{mM}$ EDTA and $2 \% \mathrm{FBS}$ ) and then incubated with FITC-CD4+ (mouse) and APC-CD8+ (mouse) antibodies for $2 \mathrm{~h}$ at $4{ }^{\circ} \mathrm{C}$ in the dark, respectively. Flow cytometry analysis was performed on a BD FACS Caliber equipped with Cell Quest software (BD Biosciences, NJ, USA).

In vivo imaging 
The permeability of blood-brain barrier (BBB) was assessed with bovine serum albumin coupled with Cy5.5 (BSA-Cy5.5) as described before(Schmitz et al., 2014). The BSA-Cy5.5 (50 mg/kg) was injected by tail vein, and imaging was performed $6 \mathrm{~h}$ after injection. The images were acquired using a IVIS Spectrum CT Imaging System (PerkinElmer, Inc. USA) with Ex/Em=680/720 nm. Myelin imaging was performed with a near-infrared dye, 3,3-diethylthiatricarbocyanine iodide (DBT), that binds to myelin and can be used to evaluated demyelination(Wang et al., 2011). DBT dye $(0.3 \mathrm{mg} / \mathrm{kg}$ ) were administered to mice by intravenous injections in the tail vein. The fluorescence signals of the mouse myelin were acquired using a IVIS Spectrum CT Imaging System (PerkinElmer, Inc. USA) 5 min post-injection of DBT with Ex/Em=745/800 nm.

\section{Histochemical analysis}

The spinal cords dissected from the spinal canal were fixed in $4 \%$ buffered paraformaldehyde for $24 \mathrm{~h}$ and then embedded in paraffin. In the following step, the standard tissue sections $(5 \mu \mathrm{m})$ were subjected to hematoxylin and eosin ( $\mathrm{H} \& \mathrm{E})$ staining and luxol fast blue (LFB) staining. Images were acquired using a digital color charge-coupled device camera and NIS-Elements imaging software. The organ sections (heart, liver, spleen, lung, and kidney) from vehicle- or piperine- treated EAE mice was subjected to H\&E analysis.

\section{Results}

\section{Piperine is a novel and potent DHODH inhibitor}

DHODH activity was determined using a chromogen 2,6-dichloroindophenol (DCIP) based reduction assay, which is stoichiometrically equivalent to the oxidation of dihydroorotate (DHO) to orotate. As shown in Figure 1A-B, piperine inhibits DHODH enzymatic activity in a dose-dependent manner and has a half maximal inhibitory concentration $\left(\mathrm{IC}_{50}\right)$ value of $0.88 \pm 0.04 \mu \mathrm{M}$, which is comparable with that of A771726, a Food and Drug Administration approved DHODH inhibitor. We then determined the inhibition mode of piperine using the Lineweaver-Burk plot. The results indicated that piperine is uncompetitive inhibitor versus the substrate DHO (Figure 1C) and a noncompetitive for CoQ (Figure 1D). In addition, using enzymatic kinetic experiments assays, we obtained affinity $(\mathrm{Ki})$ and kinetic parameters $\mathrm{K}_{\mathrm{on}}, \mathrm{K}_{\text {off }}$, and residence time (RT). As shown in Figure 1E-F, piperine exhibits long residence time $\left(\mathrm{K}_{\mathrm{on}}=1.28 \times 10^{2} \mathrm{M}^{-1} \mathrm{~s}^{-1}, \mathrm{~K}_{\mathrm{off}}=9.11 \times 10^{-5}\right.$ $\left.\mathrm{s}^{-1}, \mathrm{RT}=182.95 \mathrm{~min}\right)$ and high affinity $\left(\mathrm{Ki}=\mathrm{K}_{\mathrm{off}} / \mathrm{K}_{\mathrm{on}}=1.18 \mu \mathrm{M}\right)$ for DHODH.

\section{Piperine directly interacts with DHODH protein}

Next, we examine the interaction between piperine and DHODH by using circular dichroism (CD) spectroscopy. This technique is widely used to probe the binding of ligand to protein through analyzing the induced CD (ICD) spectrum(Micsonai et al., 2015). Purified DHODH protein was incubated with different concentrations of piperine in phosphate buffer, and detected by CD. As presented in Figure 2A, piperine induced a significant alteration of CD spectrum, indicating a changed secondary structure of DHODH protein. Considering the existing of tryptophan residues (Trp 362) in human DHODH protein, we perform an intrinsic fluorescence-quenching assay to further confirm the binding of piperine to DHODH (24). In this assay, DHODH $(20 \mu \mathrm{M})$ was incubated with varied concentrations of piperine, and the fluorescence signals were measured. As shown in Figure 1C, DHODH protein have a maximal fluorescence intensity at 337 $\mathrm{nm}$, and PIP dose-dependently induce fluorescence quenching of DHODH protein, suggesting the binding of piperine to $\mathrm{DHODH}$.

Then, thermal shift assays (TSA) was used to investigate DHODH protein thermal stabilization upon compound binding(Lo et al., 2004). DHODH protein $(5 \mu \mathrm{M})$ was incubated with various concentrations of piperine and the mixture was subjected to thermal scanning. The Melting temperature (Tm) was calculated from the Melt curve. Figure 1C shows that piperine dramatically improves the stability of DHODH protein as a reflection of the increased Tm values over $5.12{ }^{\circ} \mathrm{C}$. Furthermore, the thermodynamic parameters of the interaction of piperine and DHODH was examined using isothermal titration calorimetry (ITC). The purified recombinant human DHODH protein was titrated with piperine and the reaction heat was recorded. As shown in Figure 1D, piperine binds to DHODH with a $K_{D}$ value of $1.97 \pm 0.20 \mu \mathrm{M}$ according to the ITC titration curves, which is comparable with results of DHODH enzymatic kinetic analysis $(\mathrm{Ki}=0.71 \pm 0.01$ 
$\mu \mathrm{M})$. Put together, these results show the potent binding potency of piperine to DHODH.

\section{Piperine co-crystallizes with human DHODH}

Next, we solved the X-ray cocrystal structure of piperine bound to DHODH at a resolution of $1.98 \AA$ (PDB code: $6 \mathrm{IWU}$ ). The high-resolution structure shows that piperine is located in the hydrophobic channel formed between helix $\alpha 1$ and $\alpha 2$ (Figure 3A). This channel can be roughly divided into an exclusive hydrophobic entrance and a rather polar narrow end, and capped by a small hydrophobic pocket formed by side chains of Val134 and Val143 (Figure 3B). Thus, the amphipathic character of binding channel is a genuine pocket for potential ligands binding. Obviously, the electron density unequivocally reveals that piperine matches this amphipathic requirement well: the 1,3-benzodioxole ring located in the hydrophobic part of the channel and the n-piperidinecarbonyl of the piperine deeply buried in the polar environment. The 1,3-benzodioxole ring of piperine contacting with residues including Met 43, Val 143, Val 134, Pro 52, Phe 98, Ala 59, Leu 68, Pro 396 forms a protruding hydrophobic patch (Figure 3C). The N-piperidinecarboxaldehyde moiety of the piperine bonds to the phenolic hydroxyl group of Tyr 356 through a hydrogen bond and overlays well in the

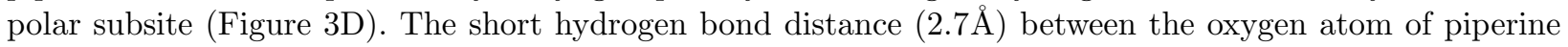
and the hydroxyl of Tyr 356 in the crystallographic structure indicates a reliable hydrogen bond interaction. DHODH have two important redox sites, one where FMN oxidizes dihydroorotate to orotate and the other where ubiquinone oxidizes FMNH2 to FMN(Liu, Neidhardt, Grossman, Ocain \& Clardy, 2000a). Piperine can occupy the narrow region in the FMNH2-ubiquinone redox site, which can impact the following DHODH activity. Polar and hydrophobic interactions were exhibited clearly in the two-dimensional view of piperine contacting with DHODH, and there is no water in the binding pocket (Figure 3E).

\section{Piperine reduces spleen lymphocytes proliferation by inhibiting DHODH}

Human DHODH is a critical enzyme for de novo pyrimidine synthesis, and it has been pharmacological targeted for the therapy of autoimmune diseases with self-reactive T lymphocytes(Goodnow, 2007). Multiple sclerosis is an immune-mediated disease and blocking autoreactive $\mathrm{T}$ cells is considered to be a promising strategy(Jelcic et al., 2018; Löffler, Fairbanks, Zameitat, Marinaki \& Simmonds, 2005). To test the immunosuppressive effects of piperine, we investigate the anti-proliferative potential of piperine by using concanavalin A (Con A)-stimulated mouse spleen lymphocytes expansion (Figure 4A). Known DHODH inhibitor A771726 was used as a positive control. As shown in Figure 4B, piperine markedly impairs ConA-induced T lymphocytes proliferation in a dose-dependent manner. Considering that DHODH is the rate-limiting enzyme in the endogenous biosynthesis of uridine monophosphate, we asked whether uridine treatment could rescue piperine-induced anti-proliferation on $\mathrm{T}$ lymphocytes. As speculated, uridine treatment completely eliminates piperine's inhibition capacity on ConA-induced lymphocytes proliferation (Figure 4B). The mixed lymphocyte reaction (MLR) is a classical model for T-cell reactivity. Similarly, we found that piperine exhibits strong suppressive activities on MLR in a dose-dependent manner, while uridine supplement significantly recovers lymphocytes proliferation (Figure 4C-D).

Then, we turn to investigate whether cellular DHODH activity is affected by piperine. Cultured Jurkat T cells were treated with piperine and cellular orotic acid was quantified by a fluorogenic probe 4-trifluoromethylbenzamidoxime(Yin, Kabashima, Zhu, Shibata \& Kai, 2017) (Figure 4E). As shown in Figure 4F, piperine treatment significantly reduces DHODH activity of Jurkat $\mathrm{T}$ cells in a dose-dependent manner with an $\mathrm{IC}_{50}$ value of $0.75 \mu \mathrm{M}$. Taken together, these results suggest that piperine reduces lymphocytes proliferation via directly inhibiting DHODH.

\section{Piperine reduces clinical score and severity of EAE}

MS is an inflammatory and autoimmune disease. We next sought to determine whether pharmacological inhibition of DHODH by piperine would attenuate experimental autoimmune encephalomyelitis (EAE), a valuable model for human MS(Dendrou, Fugger \& Friese, 2015b). For EAE induction, C57BL/6 mice were immunized with MOG35-55 followed by pertussis toxin (PTX) administration (Figure 5A). EAE mice was administered daily i.p. injections with vehicle, A771726, or piperine (10 or $30 \mathrm{mg} / \mathrm{kg}$ ) from day 7 after MOG's induction and clinical sign was evaluated using a 5-point scoring system. As shown in Figure 5B, therapy 
with piperine suppresses the manifestation of clinical symptoms over the course of the study. Meanwhile, the lower sum of scores for each individual mouse indicated a reduced disease burden for most mice in the piperine-treated group (Figure 5C). In the EAE disease progression, body weight loss is considered as a quantitative surrogate marker for disease severity(Mardiguian et al., 2013). Figure 5D shows a significant recovery in animal body weight following piperine treatment during the EAE period. This schedule is referred to as preventive treatment.

We then sought to investigate whether piperine treatment introduced at the peak of the EAE clinical symptoms could reduce the severity of disease (Figure 5E). Remarkably, piperine treatment starting at the peak of EAE (day 20) lead to an accelerated recovery from the EAE clinical signs when compared to the vehicle group (Figure 5F). Furthermore, the sum of scores for individual mouse and average body weight loss was largely improved (Figure $5 \mathrm{G}-\mathrm{H}$ ). $\mathrm{H} \& \mathrm{E}$ analysis of organs sections from vehicle- or piperine- treated EAE mice suggest that piperine is well tolerant and safe following oral dosage of $30 \mathrm{mg} / \mathrm{kg}$ every day in EAE mice (Figure S1). Taken together, these results strongly suggest that piperine alleviates the disease severity of EAE in mice as administrated in both preventive and therapeutic regimens.

\section{Piperine alleviates inflammation, blood-brain-barrier and myelin destruction}

MS has numerous pathologic hallmarks as an inflammatory and autoimmune CNS disease, such as leukocyte cells infiltration, blood-brain-barrier (BBB) and myelin destruction. Spinal cords tissues were subjected to histological analysis on day 22 after MOG's immunization. As illustrated in Figure 6A, piperine treated-group showed reduced leukocyte cell infiltrations by hematoxylin and eosin (H\&E) staining and less demyelination in white matter by Luxol fast blue (LFB) staining as compared to the vehicle-treated mice.

Blood-brain-barrier (BBB) destruction is a pathologic hallmark of EAE. We then aim to investigate the effect of piperine on BBB permeability using a fluorescent BSA conjugate probe BSA-Cy5.5. As shown in Figure $6 \mathrm{~B}$, piperine significantly reduce the accumulation of BSA-Cy 5.5 in the brain as indicated by in vivo optical imaging when compared to the vehicle treated mice, suggesting improved BBB integrity in EAE challenge. During the pathological process of MS, destructive BBB allows lymphocytes activated in the periphery to infiltrate the CNS, which evoke a local immune reaction resulting in axons and myelin damages. We next examined whether piperine treatment alleviates myelin destruction in EAE model using a myelin-binding near-infrared dye 3,3-diethylthiatricarbocyanine iodide (DBT). DBT dye $(0.3 \mathrm{mg} / \mathrm{kg})$ were intravenous injection to mice and the fluorescence signals were imaged. Figure $6 \mathrm{C}$ shows a higher fluorescent intensity as compared to the vehicle group, indicating reduced myelin destruction following piperine treatment.

We also investigate the effect of piperine on EAE-related $\mathrm{T}$ cell activation. As presented in Figure 6D-E, EAE challenge induce a significantly increase of population of $\mathrm{CD} 4^{+}$and $\mathrm{CD} 8^{+} \mathrm{T}$ cell in spleen (increased from $9.35 \pm 0.17 \%$ to $13.6 \pm 0.32 \%$ for $\mathrm{CD}^{+} \mathrm{T}$ cell and from $16.17 \pm 0.18 \%$ to $24.32 \pm 0.39 \%$ for $\mathrm{CD} 8^{+} \mathrm{T}$ cell). Remarkably, piperine treatment group $(30 \mathrm{mg} / \mathrm{kg})$ shows a significantly reduction of $\mathrm{CD} 4^{+}$population, when compared with the vehicle-treated mice. In addition, reduced CD8 ${ }^{+}$proportion was also observed following piperine administration. Taken together, these data indicate that piperine can effectively ameliorate inflammation and protect blood-brain-barrier and myelin in EAE mice.

\section{Discussion}

MS is a chronic disease of the CNS characterized by autoimmune inflammation, demyelination, and axonal damage(Comabella \& Khoury, 2012; Reich, Lucchinetti \& Calabresi, 2018). The underlying immunological disorders in MS result in a variety of neurological and autoimmune symptoms. A number of studies have revealed a critical role of $\mathrm{T}$ cells in the pathogenesis of EAE and MS makes them a promising target for drug discovery(Fletcher, Lalor, Sweeney, Tubridy \& Mills, 2010). Pyrimidine nucleotides are critical for DNA and RNA synthesis and are indispensable for the development and survival of mature T lymphocytes(Quemeneur, Gerland, Flacher, Ffrench, Revillard \& Genestier, 2003). Human DHODH catalyzes the fourth committed step in the de novo pyrimidine nucleotide biosynthetic pathway(Liu, Neidhardt, Grossman, Ocain \& Clardy, 2000a). Pharmacological intervention of pyrimidine biosynthesis by selectively inhibition of DHODH has been explored for the development of new therapeutic agents against MS. Synthetic small molecule A771726 
have been approved for the treatment of MS as an immunomodulatory drug. However, the long elimination half-life of A771726 is associated with its observed hepatotoxicity in clinical and potential teratogenicity in animal study(Garnock-Jones, 2013). More safe and effective therapeutic agents targeting DHODH are needed to benefit patient with MS.

Black pepper was widely used in traditional medicine as a natural herb for the therapy of a variety of diseases including rheumatism, pains, and fevers(Gorgani, Mohammadi, Najafpour \& Nikzad, 2017). Piperine is the predominant active constituent of black pepper and also is the most abundant alkaloid in pepper (approximately 98\%). Previous research has revealed that piperine has widely pharmacological activities, including anti-inflammatory, antiarthritic effects, antioxidant and chemopreventive activities(Bang et al., 2009; Manayi, Nabavi, Setzer \& Jafari, 2017). To the best of our knowledge, this is the first study of revealing piperine's potent protective activity in autoimmune demyelinating disease.

Piperine was widely used as a flavoring ingredient in foods and beverages manufacturers with welldocumented safety since it is first isolated from pepper in 1819(Gorgani, Mohammadi, Najafpour \& Nikzad, 2017). Using modern drug safety evaluation system, Bastaki $M$ et al organized a 90-day GLP compliant toxicity study in sprague-dawley rats with multiple doses of piperine $(5-50 \mathrm{mg} / \mathrm{kg}$ bw/day) to evaluate the safety of daily uptake of piperine in foods. Notably, no adverse effects were observed following piperine ingestion even at the highest dosage $(50 \mathrm{mg} / \mathrm{kg}$ bw/day)(Bastaki et al., 2018). Herein, we present data that piperine has the ability to interact with DHODH and reduce $\mathrm{T}$ cell proliferation via a DHODH dependent manner. We further show that piperine effectively alleviates autoimmune response in MOG-induced EAE mouse model without obvious toxicity following the oral administration of piperine $(30 \mathrm{mg} / \mathrm{kg})$. As piperine exerts its pharmacological effects in preclinical MS model through DHODH inhibition, and as piperine is a clinical stage compound with excellent safety(Quijia \& Chorilli, 2020), drug repurposing of piperine may be a valuable manipulation to benefit patients with MS.

According to the MS immunopathogenesis hypothesis, activated T cells can cross the BBB and reactive with CNS proteins, including myelin basic protein $[\mathrm{MBP}]$ and myelin oligodendrocyte glycoprotein [MOG](BarOr, Pachner, Menguy-Vacheron, Kaplan \& Wiendl, 2014). The BBB penetration of a small molecule may facilitate targeting activated $\mathrm{T}$ cells in the brain lesion. Intriguingly, piperine is capable of crossing the $\mathrm{BBB}$ as detected in an in vitro parallel artificial membrane permeability assay. Furthermore, in vivo tissue distribution assay indicated that piperine can penetrate BBB in rat (Liu et al., 2013). So, the BBB penetration of piperine may lay a foundation for its superior pharmacological activity in nervous system.

Piperine was identified as a less potent agonist of TRPV1 $\left(\mathrm{EC}_{50}=37.9 \mu \mathrm{M}\right)(\mathrm{McNamara}$, Randall \& Gunthorpe, 2005). Through a bioassay-guided isolation of the ethanol extract from the fruits of Piper longum, Ro's group have identified piperine as a moderate dual MAO-A and MAO-B inhibitor with $\mathrm{IC}_{50}$ values of 20.9 $\mu \mathrm{M}$ and $7.0 \mu \mathrm{M}$, respectively(Munier-Lehmann, Vidalain, Tangy \& Janin, 2013). Herein, we demonstrate that piperine is a potent, direct $\mathrm{DHODH}$ inhibitor with an $\mathrm{IC}_{50}$ value of $0.88 \pm 0.04 \mu \mathrm{M}$. Meanwhile, piperine effectively reduced $\mathrm{T}$ cell proliferation through DHODH inhibition in vitro, suggesting a potential role of piperine in immune function modulating. Furthermore, piperine treatment significantly reduce immune cell infiltration in central nervous tissue and resultant remission of EAE symptoms. We thereby speculate that DHODH is a main functional target of pepirine in EAE, although it remains to be investigated in the future whether other proteins were involved in the therapeutic effect of piperine in EAE.

In summary, we demonstrate that piperine regulates the activation of $\mathrm{T}$ lymphocytes through via pharmacological inhibiting DHODH and blocking de novo pyrimidine biosynthesis. Importantly, EAE disease progression was observably alleviated following oral administration of piperine in preventive and therapeutic regimens. These compelling preclinical data presented here suggest that piperine is a potential therapeutic agent for MS with a novel mechanism-of-action, and further demonstrate that DHODH is a promising therapeutic target for the treatment of MS.

\section{Acknowledgements}

This study was supported by the National Natural Science Foundation of China $(81773775,81973362$, 
81830083), Shanghai Committee of Science and Technology (18431900500, 19ZR1473500), Open Research Project of Key Laboratory of High-Incidence-Tumor Prevention \& Treatment (Guangxi Medical University), Ministry of Education. We thank Dr. Wenming Qin at SSRF beamline BL19U for assistance with data collection.

\section{Conflict of Interest}

All the authors do not have any conflicts of interest.

\section{References}

Adelman G, Rane SG, \& Villa KF (2013). The cost burden of multiple sclerosis in the United States: a systematic review of the literature. J Med Econ 16: 639-647.

Ascherio A (2013). Environmental factors in multiple sclerosis. Expert Rev Neurother 13: 3-9.

Baecher-Allan C, Kaskow BJ, \& Weiner HL (2018). Multiple Sclerosis: Mechanisms and Immunotherapy. Neuron 97: 742-768.

Bang JS, Oh DH, Choi HM, Sur BJ, Lim SJ, Kim JY, et al. (2009). Anti-inflammatory and antiarthritic effects of piperine in human interleukin 1beta-stimulated fibroblast-like synoviocytes and in rat arthritis models. Arthritis Res Ther 11: R49.

Bar-Or A, Pachner A, Menguy-Vacheron F, Kaplan J, \& Wiendl H (2014). Teriflunomide and its mechanism of action in multiple sclerosis. Drugs 74: 659-674.

Bastaki M, Aubanel M, Bauter M, Cachet T, Demyttenaere J, Diop MM, et al. (2018). Absence of adverse effects following administration of piperine in the diet of Sprague-Dawley rats for 90 days. Food Chem Toxicol 120: 213-221.

Comabella M, \& Khoury SJ (2012). Immunopathogenesis of multiple sclerosis. Clin Immunol 142: 2-8.

Dendrou CA, Fugger L, \& Friese MA (2015a). Immunopathology of multiple sclerosis. Nat Rev Immunol 15: $545-558$.

Dendrou CA, Fugger L, \& Friese MA (2015b). Immunopathology of multiple sclerosis. Nature Reviews Immunology 15: 545-558.

Diao Y, Lu W, Jin H, Zhu J, Han L, Xu M, et al. (2012). Discovery of diverse human dihydroorotate dehydrogenase inhibitors as immunosuppressive agents by structure-based virtual screening. J Med Chem 55: $8341-8349$.

Dimitrova P, Skapenko A, Herrmann ML, Schleyerbach R, Kalden JR, \& Schulze-Koops H (2002). Restriction of de novo pyrimidine biosynthesis inhibits Th1 cell activation and promotes Th2 cell differentiation. J Immunol 169: 3392-3399.

Fletcher JM, Lalor SJ, Sweeney CM, Tubridy N, \& Mills KH (2010). T cells in multiple sclerosis and experimental autoimmune encephalomyelitis. Clin Exp Immunol 162: 1-11.

Friese MA, \& Fugger L (2005). Autoreactive CD8+ T cells in multiple sclerosis: a new target for therapy? Brain 128: 1747-1763.

Garnock-Jones KP (2013). Teriflunomide: a review of its use in relapsing multiple sclerosis. CNS Drugs 27: 1103-1123.

Goodnow CC (2007). Multistep pathogenesis of autoimmune disease. Cell 130: 25-35.

Gorgani L, Mohammadi M, Najafpour GD, \& Nikzad M (2017). Piperine-The Bioactive Compound of Black Pepper: From Isolation to Medicinal Formulations. Compr Rev Food Sci F 16: 124-140. 
Goverman J (2009). Autoimmune T cell responses in the central nervous system. Nat Rev Rheumatol 9: 393.

Holland NJ, Schneider DM, Rapp R, \& Kalb RC (2011). Meeting the needs of people with primary progressive multiple sclerosis, their families, and the health-care community. Int J MS Care 13: 65-74.

Jelcic I, Al NF, Wang J, Lentsch V, Planas R, Jelcic I, et al.(2018). Memory B Cells Activate Brain-Homing, Autoreactive CD4+ T Cells in Multiple Sclerosis. Cell 175 (1): 85-100.e123.

Jennum P, Wanscher B, Frederiksen J, \& Kjellberg J (2012). The socioeconomic consequences of multiple sclerosis: a controlled national study. Eur Neuropsychopharmacol 22: 36-43.

Lassmann H, \& Bradl M (2017). Multiple sclerosis: experimental models and reality. Acta Neuropathol 133: 223-244.

Liu H, Luo R, Chen X, Liu J, Bi Y, Zheng L, et al. (2013). Tissue distribution profiles of three antiparkinsonian alkaloids from Piper longum L. in rats determined by liquid chromatography-tandem mass spectrometry. J Chromatogr B Analyt Technol Biomed Life Sci 928: 78-82.

Liu S, Neidhardt EA, Grossman TH, Ocain T, \& Clardy J (2000a). Structures of human dihydroorotate dehydrogenase in complex with antiproliferative agents. Structure 8: 25-33.

Liu SP, Neidhardt EA, Grossman TH, Ocain T, \& Clardy J (2000b). Structures of human dihydroorotate dehydrogenase in complex with antiproliferative agents. Structure 8: 25-33.

Lo MC, Aulabaugh A, Jin GX, Cowling R, Bard J, Malamas M, et al.(2004). Evaluation of fluorescence-based thermal shift assays for hit identification in drug discovery. Anal Biochem 332: 153-159.

Löffler M, Fairbanks LD, Zameitat E, Marinaki AM, \& Simmonds HA (2005). Pyrimidine pathways in health and disease. Trends Mol Med 11:430-437.

Löffler M, Jöckel J, \& Schuster G (1997). Dihydroorotat-ubiquinone oxidoreductase links mitochondria in the biosynthesis of pyrimidine nucleotides. Mol Cell Biochem 174: 125-129.

Lu WQ, Yao X, Ouyang P, Dong NN, Wu D, Jiang XW, et al. (2017). Drug Repurposing of Histone Deacetylase Inhibitors That Alleviate Neutrophilic Inflammation in Acute Lung Injury and Idiopathic Pulmonary Fibrosis via Inhibiting Leukotriene A4 Hydrolase and Blocking LTB4 Biosynthesis. J Med Chem 60: 18171828.

Manayi A, Nabavi SM, Setzer WN, \& Jafari S (2017). Piperine as a potential anti-cancer agent: a Review on Preclinical studies. Curr Med Chem 25 (37): 4918-4928.

Mardiguian S, Serres S, Ladds E, Campbell SJ, Wilainam P, McFadyen C, et al. (2013). Anti-IL-17A treatment reduces clinical score and VCAM-1 expression detected by in vivo magnetic resonance imaging in chronic relapsing EAE ABH mice. Am J Pathol 182: 2071-2081.

McLean JE, Neidhardt EA, Grossman TH, \& Hedstrom L (2001). Multiple inhibitor analysis of the brequinar and leflunomide binding sites on human dihydroorotate dehydrogenase. Biochemistry 40: 2194-2200.

McNamara FN, Randall A, \& Gunthorpe MJ (2005). Effects of piperine, the pungent component of black pepper, at the human vanilloid receptor (TRPV1). Br J Pharmacol 144: 781-790.

Micsonai A, Wien F, Kernya L, Lee YH, Goto Y, Refregiers M, et al. (2015). Accurate secondary structure prediction and fold recognition for circular dichroism spectroscopy. Proc Natl Acad Sci U S A 112: E30953103 .

Munier-Lehmann H, Vidalain PO, Tangy F, \& Janin YL (2013). On dihydroorotate dehydrogenases and their inhibitors and uses. J Med Chem 56: 3148-3167. 
Najm FJ, Madhavan M, Zaremba A, Shick E, Karl RT, Factor DC, et al. (2015). Drug-based modulation of endogenous stem cells promotes functional remyelination in vivo. Nature 522: 216.

Quemeneur L, Gerland LM, Flacher M, Ffrench M, Revillard JP, \& Genestier L (2003). Differential control of cell cycle, proliferation, and survival of primary $\mathrm{T}$ lymphocytes by purine and pyrimidine nucleotides. J Immunol 170: 4986-4995.

Quijia CR, \& Chorilli M (2020). Characteristics, Biological Properties and Analytical Methods of Piperine: A Review. Crit Rev Anal Chem 50: 62-77.

Reich DS, Lucchinetti CF, \& Calabresi PA (2018). Multiple Sclerosis. N Engl J Med 378: 169-180.

Ridderstad Wollberg A, Ericsson-Dahlstrand A, Jureus A, Ekerot P, Simon S, Nilsson M, et al. (2014). Pharmacological inhibition of the chemokine receptor CX3CR1 attenuates disease in a chronic-relapsing rat model for multiple sclerosis. Proc Natl Acad Sci U S A 111:5409-5414.

Ruckemann K, Fairbanks LD, Carrey EA, Hawrylowicz CM, Richards DF, Kirschbaum B, et al. (1998). Leflunomide inhibits pyrimidine de novo synthesis in mitogen-stimulated T-lymphocytes from healthy humans. J Biol Chem 273: 21682-21691.

Schmitz K, de Bruin N, Bishay P, Mannich J, Haussler A, Altmann C, et al. (2014). R-flurbiprofen attenuates experimental autoimmune encephalomyelitis in mice. EMBO Mol Med 6: 1398-1422.

Sykes DB, Kfoury YS, Mercier FE, Wawer MJ, Law JM, Haynes MK, et al. (2016). Inhibition of Dihydroorotate Dehydrogenase Overcomes Differentiation Blockade in Acute Myeloid Leukemia. Cell 167:171-186.e115.

T Hart BA, \& Massacesi L (2009). Clinical, pathological, and immunologic aspects of the multiple sclerosis model in common marmosets (Callithrix jacchus). J Neuropathol Exp Neurol 68: 341-355.

Wang C, Wu C, Popescu DC, Zhu J, Macklin WB, Miller RH, et al.(2011). Longitudinal near-infrared imaging of myelination. J Neurosci 31: 2382.

Wanke F, Moos S, Croxford AL, Heinen AP, Graf S, Kalt B, et al.(2017). EBI2 Is Highly Expressed in Multiple Sclerosis Lesions and Promotes Early CNS Migration of Encephalitogenic CD4 T Cells. Cell Rep 18: $1270-1284$.

Wu D, Wang W, Chen W, Lian F, Lang L, Huang Y, et al. (2018). Pharmacologic inhibition of dihydroorotate dehydrogenase induces apoptosis and differentiation in acute myeloid leukemia cells. Haematologica.

Xu X, Xu X, Liu P, Zhu ZY, Chen J, Fu HA, et al. (2015). Structural Basis for Small Molecule NDB (NBenzyl-N-(3-(tert-butyl)-4-hydroxyphenyl)-2,6-dichloro-4-(dimethylamino) Benzamide) as a Selective Antagonist of Farnesoid X Receptor alpha (FXRalpha) in Stabilizing the Homodimerization of the Receptor. J Biol Chem 290: 19888-19899.

Yin S, Kabashima T, Zhu Q, Shibata T, \& Kai M (2017). Fluorescence assay of dihydroorotate dehydrogenase that may become a cancer biomarker. Sci Rep 7: 40670.

Zhao LL, Wang YQ, Cao DY, Chen TT, Wang Q, Li YL, et al. (2015). Fragment-Based Drug Discovery of 2-Thiazolidinones as BRD4 Inhibitors: 2. Structure-Based Optimization. J Med Chem 58: 1281-1297.

Zozulya AL, \& Wiendl H (2008). The role of regulatory T cells in multiple sclerosis. Nat Clin Pract Neurol 4: 384-398.

Table 1. Data collection and refinement statistic of co-crystal structure of DHODH with piperine.

\begin{tabular}{ll}
\hline Data Set & Piperine \\
\hline $\begin{array}{l}\text { Data collection } \\
\text { Space group }\end{array}$ & P 3221
\end{tabular}




\begin{tabular}{ll}
\hline Data Set & Piperine \\
\hline Cell dimensions & \\
a,b,c $(\AA)$ & 90.41390 .413122 .699 \\
$\alpha, \beta, \gamma\left({ }^{\circ}\right)$ & $90,90,120$ \\
Resolution $(\AA)$ & $48.29-1.98$ \\
I/ I & $13.1 / 2.4$ \\
Completeness $(\%)$ & $98.85(98.72)$ \\
Redundancy & 14.5 \\
Rmerge & 0.313 \\
Refinement & \\
Resolution $(\AA)$ & $48.29-1.98$ \\
No. reflections & 38437 \\
Rwork/Rfree & $0.185 / 0.209$ \\
No. atoms & \\
Protein & 2766 \\
Ligand/ion & 21 \\
Water & 105 \\
R.m.s. deviations & \\
Bond lengths $(\AA)$ & 0.0228 \\
Bond angles $\left(^{\circ}\right)$ & 2.1337 \\
Ramachandran statistics & \\
Favoured & 349 \\
Allowed & 9 \\
Outliers & 2 \\
\hline
\end{tabular}

Figure legends

Figure 1. Piperine potently inhibit DHODH enzymatic activity

(A) Chemical structure of piperine.

(B) Dose-response curves of piperine and A771726 against human DHODH.

(C) Piperine is an uncompetitive DHODH inhibitor versus substrate DHO. Assays were performed using varied substrate concentrations $(25$ to $200 \mu \mathrm{M})$ with either DMSO $\quad\left(^{*}\right), \quad 1.0 \quad \mu \mathrm{M} \quad()$ or $\quad 3.0 \quad \mu \mathrm{M} \quad\left({ }^{*}\right.$ piperineasmentionedinmethods.Lineweaver -Burkplotwasusedtorevealtheinhibitioncharacteristicsof piperineagainstDHODH.

(D) piperine is noncompetitive inhibitor for CoQ0. The enzymatic reactions mixture contains the varied CoQ0 (25 to $200 \mu \mathrm{M})$ with either DMSO $(*), 1.0 \mu \mathrm{M}()$ or $3.0 \mu \mathrm{M}\left({ }^{(}\left({ }^{\prime}\right.\right.$ piperine.Lineweaver -BurkplotswereusedtorevealtheinhibitionmodeofpiperineagainstDHODH.

(E) Enzymatic kinetic curves of DHODH with a series of concentrations of piperine from 0.025 to $50 \mu \mathrm{M}$.

(F) Analysis of the enzymatic kinetic curves shown in (E) generated the following kinetic parameters and affinity for DHODH: $\mathrm{K}_{\mathrm{on}}=1.28 \times 10^{2} \mathrm{M}^{-1} \mathrm{~s}^{-1}, \mathrm{~K}_{\mathrm{off}}=9.11 \times 10^{-5} \mathrm{~s}^{-1}$ and residence time (RT) $=182.95 \mathrm{~min}$, as well as $\mathrm{Ki}=\mathrm{K}_{\mathrm{off}} / \mathrm{K}_{\mathrm{on}}=0.71 \pm 0.01 \mu \mathrm{M}$, respectively. Results are the mean \pm SEM of three independent experiments.

Figure 2. Piperine directly bind to DHODH protein

(A) Representative data of piperine binding to DHODH detected by CD. Purified DHODH protein $(20 \mu \mathrm{M})$ was incubated with $10 \mu \mathrm{M}$ PIP and subjected to CD spectroscopy analysis.

(B) Binding of piperine to DHODH was determined by intrinsic fluorescence quenching assay. DHODH protein $(20 \mu \mathrm{M})$ was treated with increasing concentrations of piperine $(10 \mu \mathrm{M}$ to $50 \mu \mathrm{M})$ for $30 \mathrm{~min}$. The 
fluorescence emission $(290-500 \mathrm{~nm}$ ) was recorded with an excitation wavelength of $280 \mathrm{~nm}$. The dashed line indicates the fluorescence spectrum of piperine $(10 \mu \mathrm{M})$.

(C) Thermal shift assay reveasl that piperine significantly stabilizes DHODH and results in a thermal shift over $5.12^{\circ} \mathrm{C}$ (molar ratio $\left.1: 20\right)$.

(D) Thermodynamic characterization of DHODH-piperine interactions by ITC. DHODH protein was titrated with piperine in ITC buffer as mentioned in methods. Binding curves were fitted as a single binding event using MicroCal origin software package. Data are presented as mean \pm SEM of three independent experiments

\section{Figure 3. Piperine co-crystallize with DHODH}

(A-B) The overall structure of DHODH in complex with piperine. Structure of DHODH-piperine complex, with protein backbones shown as a gray surface. Superposition of the PIP molecule (pink) is shown in stick representation. The residues of $\alpha 1$ and $\alpha 2$ (orange), involved in hydrophobic contacts with PIP, is exhibited as carton.

(C-D) Ubiquinone-binding patch of DHODH with PIP. The 2Fo-Fc electron density maps of PIP in DHODH is contoured at $1.5 \sigma$. Dashed lines indicate potential hydrogen bonds. The residues involved in ligand binding are labelled with yellow sticks.

(E) Two-dimensional view of PIP interactions with DHODH. Residues accounting for polar and hydrophobic interactions are shown in blue and green boxes.

Figure 4. Piperine inhibits mouse spleen cell proliferation via inhibition of DHDOH.

(A) Schematic diagram of concanavalin-A (Con A) induced spleen cell activation.

(B) Spleen cells isolated from naive C57BL/6 mice were cultured with $10 \mu \mathrm{g} / \mathrm{mL}$ of Con A in the presence of various concentration of piperine. After culture for 3 days, cell viability was determined using MTT assay. Each value indicates the mean $\pm \mathrm{SD}$ of three independently experiments.

(C) Schematic diagram of mixed lymphocyte reaction (MLR) assay.

(D) Single-cell suspensions of spleens from C57BL/6 and BALB/C were prepared as described in methods section. Each set of $2 \times 10^{5}$ cells were cultured in 96 -well cell culture plates with a serial dilution of piperine. Cell proliferation was evaluated after 4 days by MTT as described above. Each value indicates the mean \pm $\mathrm{SD}$ of three independently experiments.

(E) Principle of the fluorescence-based assay for cellular DHODH activity. DHO was firstly enzymatic catalyzed to orotic acid by cellular DHODH and orotic acid was further detected by a fluorescence probe 4-trifluoromethyl-benzamidoxime (4-TFMBAO).

(F) The enzymatic activities of DHODH in Jurkat T cells treated with a series of concentrations of piperine were determined by fluorescence assay as described in (E).

Figure 5. Piperine reduces clinical score and severity of EAE

(A) Schematic diagram of EAE model with piperine treatment beginning at day 10.

(B) Daily mean EAE clinical score of C57BL6 mice $(\mathrm{n}=8)$ on preventive treatment with vehicle, piperine $(10 \mathrm{mg} / \mathrm{kg}$ and $30 \mathrm{mg} / \mathrm{kg}$ ), or A771726 (10 mg/kg). Drugs was administered through daily i.p. injection at day 10 after EAE induction. Data are shown as mean +- SEM and Student's t-test is performed, ${ }^{* *} \mathrm{P}<0.01$, $* * * \mathrm{P}<0.001$.

(C) The sum of EAE scores for each individual mouse in different groups in $\mathbf{b}$. Data are shown as mean +SEM and Student's t-test is performed, ${ }^{* *} \mathrm{P}<0.01,{ }^{* * *} \mathrm{P}<0.001$.

(D) The body weight was recorded daily during the EAE course (day12-day28). Data are shown as mean +- SEM and Student's t-test is performed, ${ }^{* *} \mathrm{P}<0.01,{ }^{* * *} \mathrm{P}<0.001$. 
(E) Schematic diagram of EAE model with piperine treatment initiating at day 20.

$(\mathrm{F})$ Daily mean EAE clinical score of C57BL6 mice $(\mathrm{n}=8)$ on therapeutic treatment with vehicle, piperine $(30 \mathrm{mg} / \mathrm{kg})$, or A771726 (10 mg/kg). Drugs was administered through daily i.p. injection at day 20 after EAE induction. Data are shown as mean +- SEM and Student's t-test is performed, ${ }^{* *} \mathrm{P}<0.01$.

(G) The sum of EAE scores for each individual mouse in different groups in (F). The piperine-treated mice showed a notably decrease in the total scoring. Data are shown as mean +- SEM and Student's t-test is performed, ${ }^{*} \mathrm{P}<0.05,{ }^{* * *} \mathrm{P}<0.001$.

(H) The body weight was recorded daily during the EAE course (day14-day30). Data are shown as mean +- SEM and Student's t-test is performed, ${ }^{*} \mathrm{P}<0.05$, ${ }^{* * *} \mathrm{P}<0.001$.

\section{Figure 6. Piperine relieves inflammation and myelin destruction in EAE model}

(A) Representative histological images of spinal cord sections from piperine- or vehicle- treated EAE mice in preventive model. The sections were prepared on day 22 after MOG's immunization. Hematoxylin and eosin (H\&E) staining for assessing of inflammation (left) and luxol fast-blue (LFB) staining for assessing of demyelination (right).

(B) BBB disruption was imaged using BSA-Cy5.5 at Ex/Em=680/720 nm (left). Mice were i.v. injected with BSA-Cy5.5 $(50 \mathrm{mg} / \mathrm{kg})$ and subjected to fluorescence imaging at $6 \mathrm{~h}$ post-injection. Quantitative results of total fluorescent counts of BSA-Cy5.5 in regions of interest (right). Values are represented as mean +SEM $(\mathrm{n}=3)$. Data are shown as mean +- SEM and Student's t-test is performed, ${ }^{*} \mathrm{P}<0.05$.

(C) Myelin imaging was performed with a near-infrared dye, 3,3-diethylthiatricarbocyanine iodide (DBT) (left). DBT dye $(0.3 \mathrm{mg} / \mathrm{kg})$ were administered to mice by i.v. injections into the tail vein. The fluorescence imaging of the mouse myelin was acquired 5 min post-injection of DBT with Ex/Em=745/800 $\mathrm{nm}$. Quantitative results of fluorescent intensity of DBT in mice (right). Values are represented as mean +- SEM $(\mathrm{n}=3)$. Data are shown as mean +- SEM and Student's t-test is performed, ${ }^{*} \mathrm{P}<0.05$.

(D-E) Flow cytometry analysis of T lymphocytes. Splenocytes were incubated with APC-CD8 ${ }^{+}(\mathrm{A})$ and FITC-CD4 $^{+}(\mathrm{B})$ antibodies for $2 \mathrm{~h}$ at $4 \mathrm{degC}$ in the dark, respectively. Flow cytometry analysis was performed on a BD FACS Caliber equipped with Cell Quest software (BD Biosciences, NJ, USA). Quantitative data of $\mathrm{CD}^{+}$and $\mathrm{CD} 4^{+}$population were presented in right, respectively. Data are shown as mean +- SEM and Student's t-test is performed, ${ }^{* * *} \mathrm{P}<0.001$. 
Figure 1

A

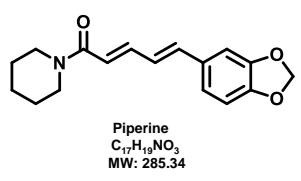

C

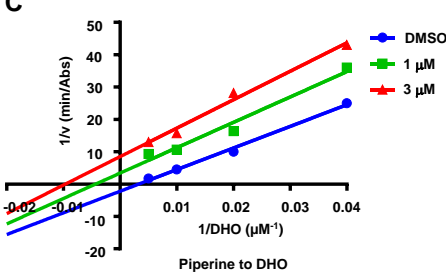

E

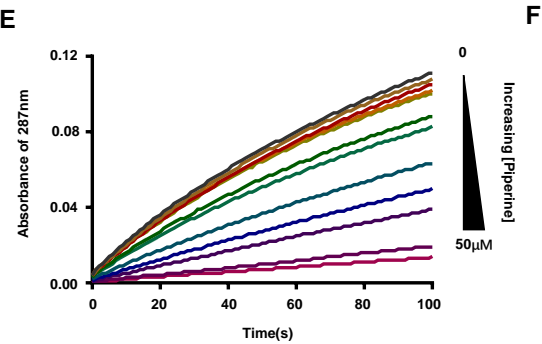

B

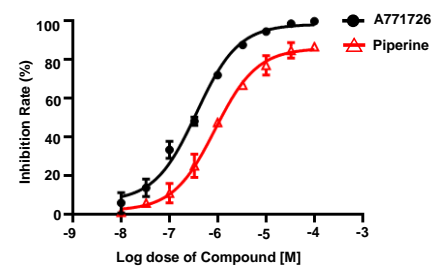

D
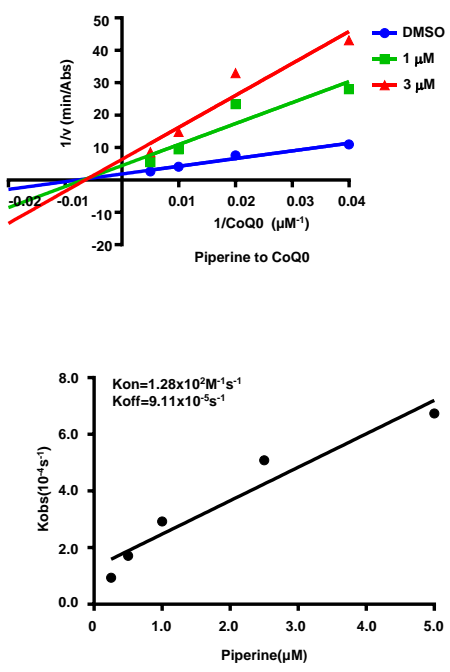

\section{Hosted file}

Figures.pdf available at https://authorea.com/users/298752/articles/428097-natural-productpiperine-alleviates-experimental-allergic-encephalomyelitis-in-mice-by-targetingdihydroorotate-dehydrogenase 